\title{
Correspondence
}

\section{Mothers' Comfort with Screening Questions}

To the Editor: The recent article by Zink et al, "Mothers' Comfort with Screening Questions about Sensitive Issues, Including Domestic Violence," 1 provides valuable clinical information regarding the difficulty of screening for domestic violence (DV) in mothers who present to the health care setting with their children. This represents the first study to quantify and qualify patients' comfort with general DV screening questions for mothers presenting alone and with their children. However, the results of this study do not adequately support routine DV screening of mothers with children present.

As the authors suggest, mothers should be screened for intimate partner violence alone whenever possible, but a clinician may employ general, less graphic, screening questions even when children are present. Not surprisingly, mothers reported more comfort answering sensitive screening questions while alone than while in the presence of their children. However, their comfort level remained reasonably high $(81.2 \%$ for general DV questions) even for screening with children present. Therefore, if a clinician wishes to implement universal screening, then the general questions used in this study at least satisfy the need for appropriateness and comfort in the presence of minors.

However, because fewer than $10 \%$ of the 200 patients studied actually had their children with them at the time of screening, comfort ratings might have been overly optimistic. Reported comfort levels alone and with children might differ according to whether or not children actually were there at the time of screening. The comfort rating for screening with children might be reduced further if mothers had rated their comfort with the screening questions in a setting in which their children were present.

In addition, the general DV screening questions used in this study have not been validated as well as more direct screening questions. ${ }^{2,3}$ These indirect, less intrusive questions might not be sensitive enough to detect DV. In fact, known victims in this study did not report significantly lower comfort ratings for screening in front of children. Evidently women who had not experienced DV were more uncomfortable with the screening process in the presence of their children than those who had been victims. This might be a case in which comfort does not translate into more reliable disclosure of DV after all.

In the absence of clear evidence to support universal screening, ${ }^{4}$ perhaps we should not try to screen women in front of their children. Patient and public education including resource awareness can create opportunities for further exploration when women are able to return to their physicians alone. Clinicians who wish to screen even when children are present can utilize confidential written questionnaires with more direct and better vali- dated questions. Positive responses can be answered by a discreet referral or an invitation to a return visit without family.

Latina mothers in this study reported significantly more discomfort with DV screening questions than Caucasians. More reliable and culturally sensitive ways in which to screen this population for DV should be explored. The authors also admit to sample and enrollment biases. I suggest repeating this study in a live clinical setting.

This study offers helpful insight into the dynamics of screening DV in patients who present to care with children. However, screening with general questions might be inadequate and unnecessary in this context. It might be more productive to educate all our patients about this important issue and to employ the best validated screening instruments in select physician-patient encounters.

S. Lindsey Clarke, MD, FAAFP

Self Regional Healthcare, Ware Shoals Center for

Family Medicine

Greenwood, SC

\section{References}

1. Zink T, Levin L, Wollan P, Putnam F. Mother's comfort with screening questions about sensitive issues, including domestic violence. J Am Board Fam Med 2006;19:358-67.

2. Brown JB, Lent B, Schmidt G, Sas G. Application of the woman abuse screening tool (WAST) and WAST-short in the family practice setting. J Fam Pract 2000;49:896-903.

3. Sherin KM, Sinacore JM, Li X, Zitter RE, Shakil A. HITS: a short domestic violence screening tool for use in a family practice setting. Fam Med 1998;30:508-12.

4. U.S. Preventive Services Task Force. Screening for family and intimate partner violence. Ann Intern Med 2004;140: $382-6$.

doi: 10.3122/jabfm.2007.01.060163

\section{Response to Dr. S. Lindsey Clarke}

To the Editor: We appreciate the comments regarding our article and agree with the authors that not asking the sensitive questions in front of the children in our study was not ideal. ${ }^{1}$ Research in the area of violence is difficult due to the nature of the topic and our Institutional Review Board did not allow us to have the children present for the interview. Despite this limitation, the study showed that mothers' comfort with the general domestic violence questions was no different from their comfort with alcohol and substance abuse questions and had more comfort than with questions about depression and sexual risk. This should give providers some reassurance about asking about domestic violence.

As the authors suggested, the sensitivity and specificity with the general questions is less than more direct questions. ${ }^{2}$ However, the general questions may be useful to begin a conversation. Recent work by McMillan et 